\title{
UNCONVENTIONAL RUBBER NETWORKS: CIRCUMVENTING THE COMPROMISE BETWEEN STIFFNESS AND STRENGTH
}

\author{
C. M. ROLAND* \\ Naval Research Lab, Chemistry Division, Code 6120, Washington, DC 20375-5342
}

RUBBER CHEMISTRY AND TECHNOLOGY, Vol. 86, No. 3, pp. 351-366 (2013)

\begin{abstract}
The failure properties of rubbery networks exhibit a maximum as a function of cross-link density or modulus. To avoid excess creep, elastomers are usually formulated such that their state of cure falls past this maximum, which means there is an inevitable compromise between modulus and failure properties (stiffness and strength). This review describes various approaches to circumventing the problem by the use of unconventional network structures. The obtainable improvements in mechanical properties can be substantial (e.g., threefold increases or greater in strength), although these methods entail additional processing steps or the use of blends and may affect the hysteresis of an elastomer; thus, their practical utility cannot be assessed a priori. [doi:10.5254/rct.13.86988]
\end{abstract}

\section{CONTENTS}

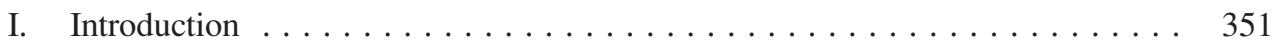

A. Interpenetrating Polymer Networks $\ldots \ldots \ldots \ldots \ldots \ldots \ldots \ldots \ldots \ldots$

B. Double Networks . . . . . . . . . . . . . . . . . . . . . . . . . . . . . . 354

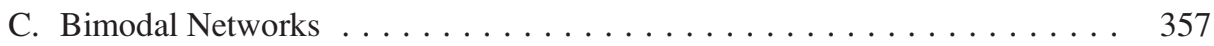

D. Miscible Blends with Components Having Different Cross

Link Densities . . . . . . . . . . . . . . . . . . . . . 358

E. Deswollen Networks . . . . . . . . . . . . . . . . . . . . . . . 362

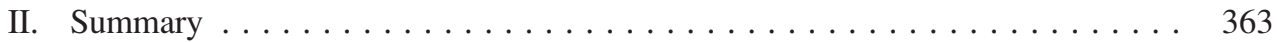

III. Acknowledgement . . . . . . . . . . . . . . . . . . . . . . . . . 364

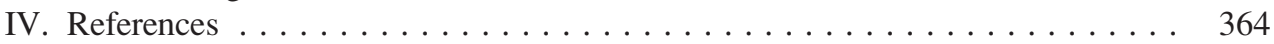

\section{INTRODUCTION}

The mechanical properties of rubbery networks do not depend strongly on chemical structure, which means that elastomers in general have very similar mechanical behavior, at least through moderate strains. Failure properties such as the strength and fatigue life can vary among polymer types, due to, for example, the effect of strain crystallization, and may depend on the type of cross-links. The most direct method of controlling failure properties is to change the concentration of cross-links. It is well known that these properties pass through a maximum versus cross-link density, reflecting the countervailing effects on network integrity and embrittlement. This is illustrated Figure $1,{ }^{1}$ showing the tensile strength of four natural rubber compounds plotted versus the modulus at $100 \%$ extension. The practically useful range of cross-linking falls past the maximum, which means that in general, elastomer failure properties decrease with increasing modulus. As seen in Figure 1, for a given degree of crosslinking, labile cross-links such as polysulfidic linkages and ionic bonds provide better properties than carbon-carbon covalent bonding, because bond dissociation under stress allows network chains to change their topology and alleviate local overstressing. For this reason, ionomers, which often have an inorganic salt grafted to the chains, can exhibit very high levels of strength and extensibility. ${ }^{1-3}$ Of course, when formulating a rubber compound, 


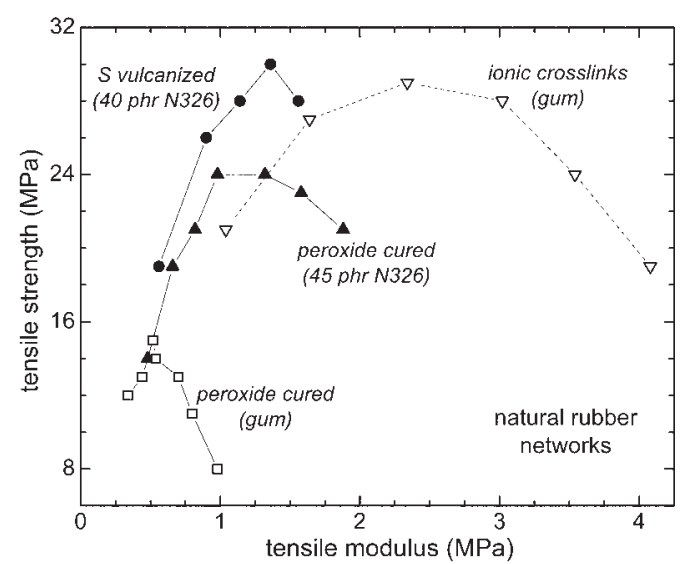

FIG. 1. - Tensile strength as a function of modulus (measured at nominal strain rates of $0.1 \mathrm{~s}^{-1}$ ) for natural rubber (NR) elastomers with cross-links having different degrees of mechanical lability, both filled (solid symbols) and unfilled (open symbols). ${ }^{1}$ Failure properties go through a maximum as a function of the degree of cross-linking.

consideration is given to a range of properties beyond stiffness and strength, for example, hysteresis. ${ }^{4}$ The focus of this review is how, for a given cross-link density and type, the failure properties can be altered by changing the nature of the network structure. Five network architectures are discussed: interpenetrating polymer networks (IPNs), double networks, bimodal networks, blends of miscible elastomers with different component cross-linking, and deswollen networks. Note there is some overlap among these network types, for example, IPNs and blend networks. Also, the nomenclature is not standardized; for example, hydrogel double networks ${ }^{5}$ are not the same as the material commonly referred to in the rubber literature as double network elastomers. The effect of cross-link type - carbon-carbon bonds, sulfidic linkages, ionic cross-links, slide-ring networks ${ }^{6}-$ are not considered herein, although they represent another obvious route to better elastomeric properties.

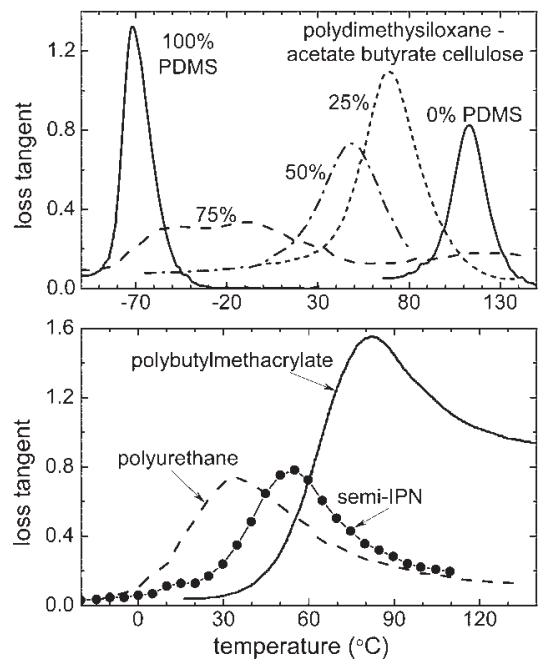

FIG. 2. - Dynamic mechanical loss tangent for interpenetrating networks of polydimethylsiloxane and acetate butyrate cellulose; the concentration by weight of the former is indicated. ${ }^{17}$ For most compositions, the phase morphology is sufficiently fine to yield only one glass transition. 


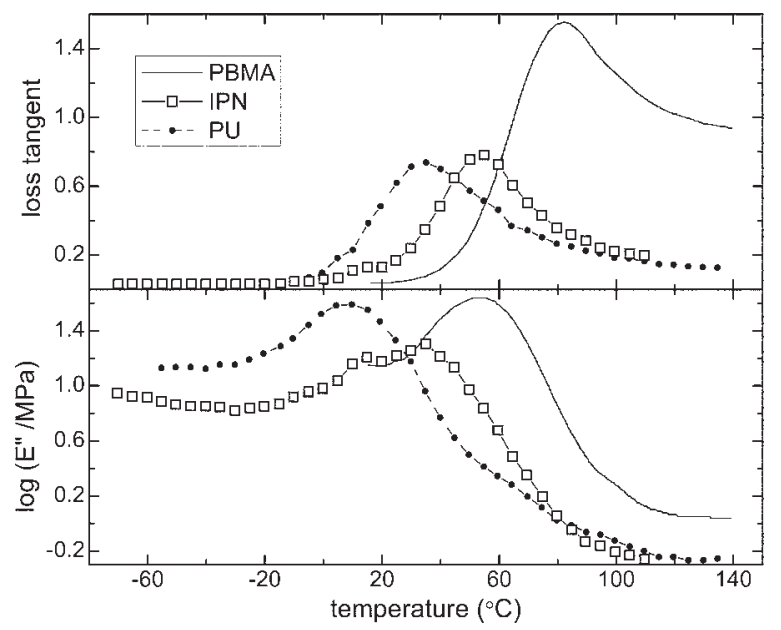

FIG. 3. - Temperature dependence of the mechanical loss tangent of a semi-IPN of equal concentrations of polybutylmethacrylate and polyurethane, along with data for the corresponding neat components. ${ }^{18}$ The behavior is consistent with a homogeneous phase morphology.

\section{A. INTERPENETRATING POLYMER NETWORKS}

The term interpenetrating polymer network refers to a method of network preparation in which polymerization and cross-linking are carried out on two components to obtain co-continuous, interlocking networks (catenanes). ${ }^{7-9}$ This co-continuity relies on kinetic retention of an initially homogenous mixture, with the formation of a network precluding phase segregation of the thermodynamically immiscible polymers. ${ }^{10,11}$ Generally, although gross phase segregation is avoided, homogeneity on the segmental level is not achieved.

IPNs can be made simultaneously or sequentially. The former involves the polymerization and cross-linking of premixed monomers or linear prepolymers. The increasing molecular weight of the components during the reaction reduces the combinatorial entropy, tending to induce phase separation; this is countered by the slower dynamics, with the spatial extent of the segregation inhibited by the cross-linking. To synthesize a sequential IPN, ${ }^{12-14}$ a network is swollen in monomers, which are then polymerized and cross-linked. The latter can be done rapidly using radiation. Phase separation is more extensive for sequential IPNs, but co-continuity has still been reported. Semi-IPN refers to IPNs in which only one component is cross-linked. ${ }^{15,16}$ The glass transition temperature, $T_{g}$, of an IPN is intermediate to those of the pure components, as shown in Figure 2 for an IPN ${ }^{17}$ and Figure 3 for a semi-IPN. ${ }^{18}$ The phase morphologies are not homogeneous, but the domains are small, so that properties such as the segmental dynamics tend to be averages. Because $T_{g}$ is size dependent for domain sizes on the order or smaller than the cooperativity length scale, changes in the glass transition temperatures of the components do not necessarily indicate mixing on the segmental level. If there is a distribution of phase sizes, very broad glass transitions result. This property of IPNs makes them attractive for acoustic damping and vibration isolation applications, where broadband and temperature-insensitive damping are desired. ${ }^{19,20}$

The process of synthesizing an IPN assumes there is no interference between the polymerization and cross-linking reactions; however, this is not necessarily the case. Grafting between the components can occur, which affects both the morphology, by inhibiting phase separation, and the properties. ${ }^{7,21-24}$ The interlocked networks of an IPN can confer greater strength than a phase-separated blend, yielding properties that are not merely additive in the component properties. Recently, a model incorporating the role of damage accumulation to the mechanical 


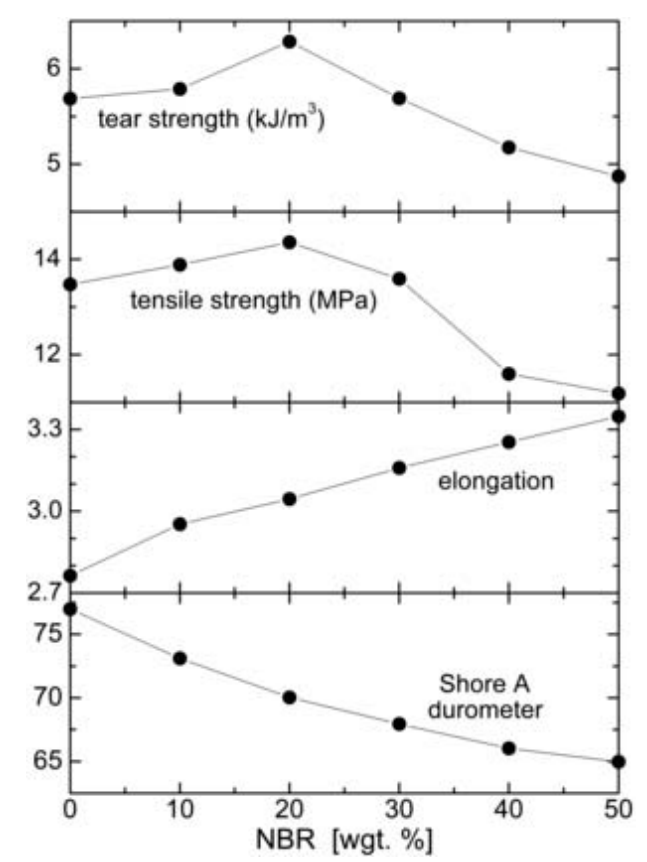

FIG. 4. - Mechanical properties of an interpenetrating polymer network of nitrile rubber and poly(vinylidene fluoride-cohexafluoroproylene). ${ }^{24}$ Failure properties exhibit a maximum versus composition.

properties of IPNs has been proposed..$^{25}$ The potential for better properties is illustrated ${ }^{23}$ in Figure 4 for an IPN of a fluoroelastomer and nitrile rubber. With increasing content of the latter, the hardness decreases and the failure strain increases, but the failure properties go through a maximum versus composition.

An interesting approach to IPNs that incorporates a feature of double networks (discussed below) is the polymerization and cross-linking of monomers absorbed into a stretched network. ${ }^{26}$ The second network is formed under compression, so that at mechanical equilibrium, the components of the IPN are mechanically balanced. The method shows promise for actuators, potentially functioning as artificial muscles.

\section{B. DOUBLE NETWORKS}

A special type of IPN is a double network rubber, in which the chain segments belong to two networks distinguished by their different orientations. A double network is formed by cross-linking the material twice, the second time while the elastomer is in a deformed state (usually tension). Because the properties of an elastomeric network depend not only on the cross-link density but also on the distribution and orientation of the chains, the properties of double networks can be quite different from those of the corresponding single network at equal degrees of cross-linking, including greater stiffness and strength. Early studies of the effect of trapped entanglements on the modulus of elastomers employed double networks. ${ }^{27,28}$ Double networks can arise spontaneously via chain scission ${ }^{29,30}$ (a process referred to as chemical stress relaxation ${ }^{31}$ ), strain-induced crystallization, ${ }^{32,33}$ or as a result of reinforcing fillers. ${ }^{34}$ Liquid crystal elastomers prepared by cross-linking the material in the nematic state are another type of double network. ${ }^{35,36}$

Analysis of double networks relies on the independent network hypothesis, ${ }^{37-40}$ which assumes there is no coupling of the behavior of the component networks, whereby the observed 


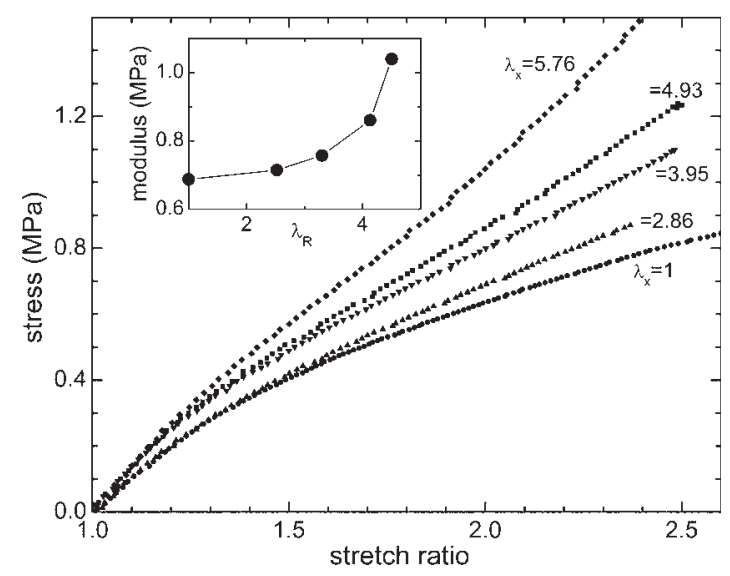

FIG. 5. - Engineering stress versus stretch ratio for double networks of polyisoprene having the indicated strain during the second cross-linking. ${ }^{49}$ The total cross-link density was the same for all samples, but there is a systematic increase in stiffness with cross-linking strain, which corresponds to increasing residual strain. The stress at $100 \%$ strain is shown in the inset.

mechanical response is the sum of the individual contributions. The equilibrium configuration of each network corresponds to that existing during network formation, so the strain energy of a double network is

$$
W_{D N}(\lambda)=W_{1}(\lambda)+W_{2}\left(\lambda / \lambda_{X}\right)
$$

in which the numerical subscripts refer to the first and second network, and $\lambda_{X}$ is the stretch ratio during the second cross-linking (the initial network being formed in the unstressed, isotropic state). The stretch ratio in Eq. 1 is referenced to the undeformed network; hence, $\lambda=\lambda_{1}$ and $\lambda_{2}=\lambda / \lambda_{X}$. The residual strain at zero stress of the double network represents the state for which the forces from the initial network, which is subsequently extended, are balanced by those from the second, compressed network. Although the stress in Eq. 1 is given simply as the sum of the component stresses, modeling of the mechanical behavior is still limited by the poor ability of elasticity theories to describe simultaneously tensile and compressive strains. ${ }^{41,42}$

The orientation of the network chains gives rise to anisotropic mechanical properties. ${ }^{43-48}$ Generally, the modulus increases parallel to the strain during cure, with negligible changes in the transverse direction. This modulus enhancement is illustrated in Figure 5 for a series of elastomers having the same total cross-link density ${ }^{49}$ but with different strains during the second cross-linking. For double networks reinforced with carbon black, lower electrical conductivity ${ }^{50}$ and suppression of the Payne effect ${ }^{51}$ have been observed, both consistent with deflocculation of the filler particles by the strain imposed during curing.

Although the experimental results are scattered, ${ }^{43-48}$ the strength of a double network appears to be marginally lower than that of the corresponding single network at equal cross-link density. Because their modulus is higher, the strength of a double network is higher when the comparison is made at equal modulus; thus, double networks circumvent the compromise between the stiffness and strength of conventional elastomers. Moreover, double networks of strain-crystallizing rubbers having substantially larger fatigue lifetimes (Figure 6). ${ }^{44,52}$ This appears to result from retention of crystallinity through the minimum of the strain cycle, ${ }^{52}$ analogous to the greater fatigue life of crystallizing rubbers subjected to nonrelaxing strain cycles. ${ }^{53}$

According to the stress optical law, the birefringence, $\Delta n$, is proportional to the stress, $\sigma$,

$$
\Delta n \equiv n_{x}-n_{y}=n_{x}-n_{z}=C \sigma_{x}
$$




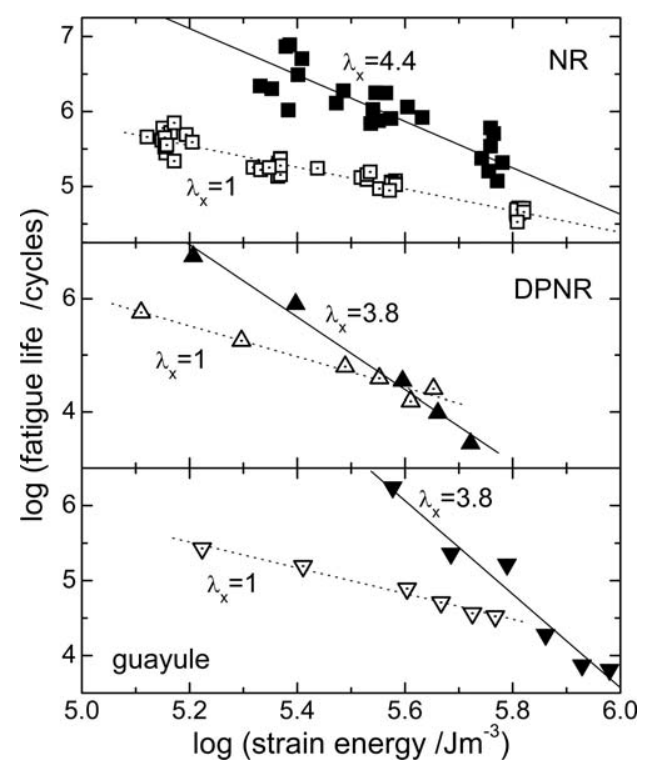

FIG. 6. - Fatigue life as a function of the recoverable strain energy for single (hollow symbols) and double (solid symbols) networks of Hevea natural rubber (squares), deproteinized natural rubber (triangles), and guayule rubber (inverted triangle), measured in tension parallel to the curing strain. ${ }^{44,52}$ The order of magnitude increases for the double networks are in contrast to the behavior of non-strain-crystallizing elastomers.

where $C$ is the stress-optical coefficient, a material constant determined by the chemical structure of the repeat unit. The principal components of the refractive index, $n_{x}, n_{y}$, and $n_{z}$, become unequal upon orientation, causing birefringence. As illustrated in Figure 7, double networks are birefringent in the absence of external stress, ${ }^{47,49}$ contrary to Eq. 2 . Such stress-free birefringence can be rationalized from constraint models of rubber elasticity, ${ }^{35}$ which predict that the ratio of the birefringence to the true stress varies with strain. This means that the birefringence of the two component networks, although having the opposite sign, do not have the same magnitude; thus, their contributions to $\Delta n$ do not cancel. ${ }^{49}$

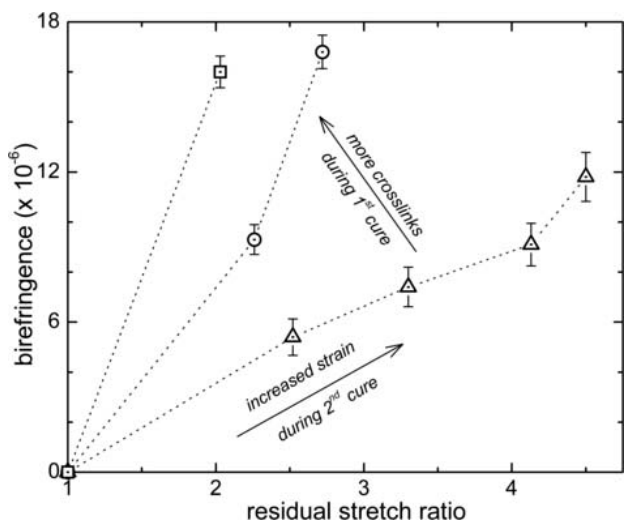

FIG. 7. - Birefringence versus residual strain for double networks at mechanical equilibrium (zero external stress); all materials had the same total cross-link density. ${ }^{49}$ Different amounts of residual strain, as reflected in the magnitudes of $\Delta n$, are obtained for the same residual strain, indicating that parameter does not uniquely define the double-network structure. 


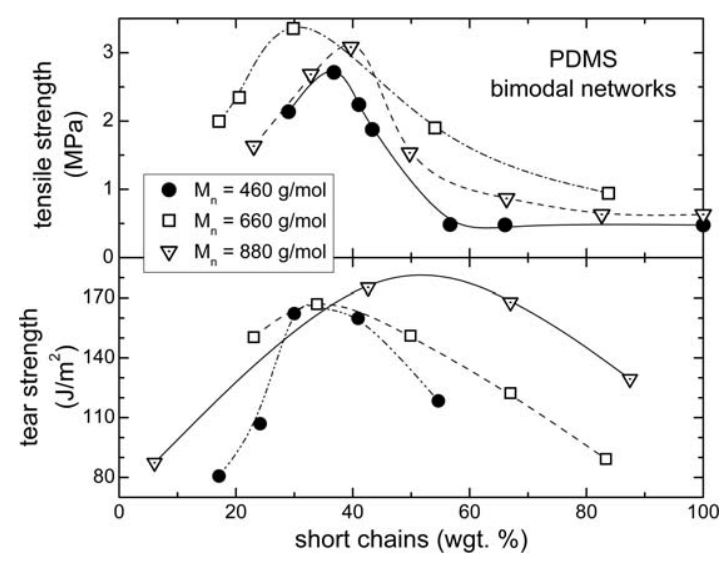

FIG. 8. - Tensile and tear strength of PDMS bimodal networks as a function of the concentration of short chains (having number average molecular weight as indicated). ${ }^{62,63}$ For the long chains, $M_{n}=21,300 \mathrm{~g} / \mathrm{mol}$. The maxima correspond to concentrations of short chains exceeding $90 \%$ by mole.

\section{BIMODAL NETWORKS}

A bimodal network is an elastomer in which most network chains are very short and the remaining are very long; they are prepared by end-linking a mixture of low- and high-molecularweight precursor chains. ${ }^{54}$ Scattering experiments show that some inhomogeneity exists in bimodal networks, on the scale of the network mesh. ${ }^{55}$ There is also indication in polydimethysiloxane (PDMS) networks of segregation of short and long chains ${ }^{56-58}$; such clustering is likewise observed in computer simulations. ${ }^{59}$ When the short chains are in excess, the mesh size of a bimodal network is essentially the same as for unimodal networks composed of the same short chains ${ }^{60}$; that is, a low concentration of long network strands has a negligible effect on the average mesh size. For bimodal networks of primarily long chains, this correlation length reflects the distance between entanglements, rather than the length between the covalent network junctions. ${ }^{60}$

Bimodal networks are attractive materials for fundamental studies of rubber elasticity, given the regularity of their structure in comparison to the random nature of conventional cross-linking.

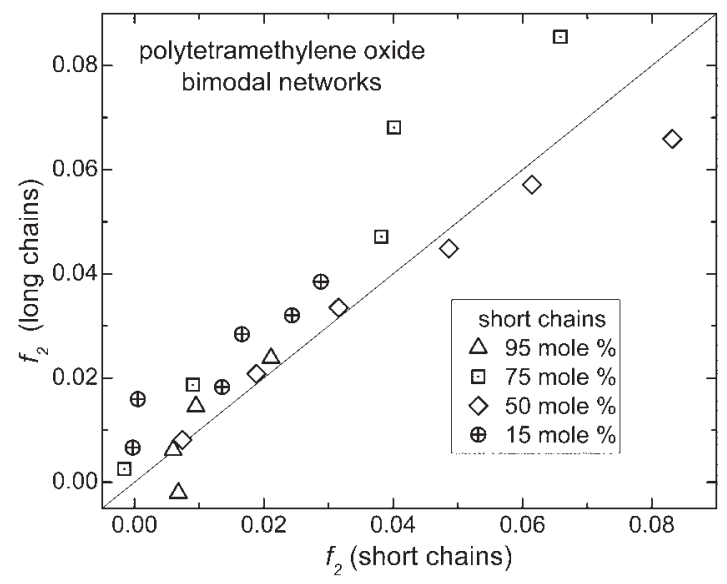

FIG. 9. - Hermans orientation function (second-order Legendre polynomial) for the long chains versus that for the short chains in polytetramethylene oxide bimodal network; the concentration of short chains is indicated. ${ }^{67}$ The data indicate nearly equal orientation (denoted by the solid line). 


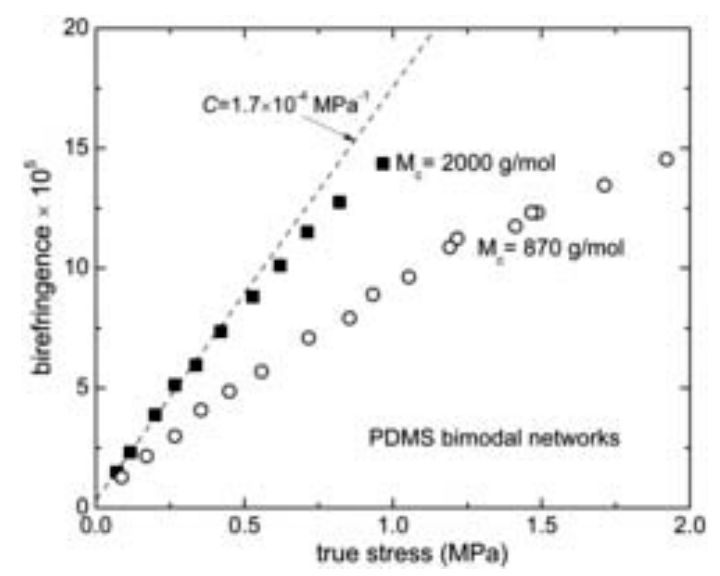

FIG. 10. - Optical birefringence of polydimethylsiloxane end-linked bimodal networks having 99\% short chains of the indicated molecular weight. ${ }^{70}$ The stress optic law is denoted by the dashed line having the indicated value of the stress optical coefficient. Deviations, presumably reflecting finite chain extensibility, are observed at lower strain for the short network chains.

Bimodal networks have been used to study the contribution of entanglements to the elastic modulus. ${ }^{61}$ The practical appeal of bimodal networks is their potential for substantially higher elongation and strength ${ }^{62-64}$ (Figure 8). Such behavior is contrary to classic theories of rubber elasticity, which assume failure occurs due to rupture of the shortest network chains ${ }^{65}$; this implies that the short chains would weaken the network. The improved properties of bimodal networks have been ascribed to a synergy between the high modulus of the short chains and the extensibility of the longer ones (a so-called delegation of responsibilities ${ }^{54,66}$ ). However, infrared dichroism measurements on deuterium-labeled polytetramethylene oxide bimodal networks reveal the orientation of the long chains to be not much different than that of the short chains (Figure 9), ${ }^{67}$ which is at odds with the putative mechanism for enhanced performance. Improved properties in bimodal networks require short chains (molecular weights of a few hundred Daltons) that are as much as a factor of 100 shorter than the long chains. ${ }^{67}$ The data in Figure 8 exhibit maxima, indicating that the optimum number density of the short chains is very high, corresponding to weight fractions in the $30-50 \%$ range. Note that the converse, a small weight fraction of long chains, also improves toughness, although such materials are not elastomeric. ${ }^{68,69}$

Figure 10 shows $^{70}$ that bimodal networks having lower-molecular-weight short chains deviate from Gaussian network behavior (proportionality between true stress and birefringence) at smaller strains. Experiments on polyethylene oxide ${ }^{71}$ and polytetramethylene oxide ${ }^{72}$ also show a greater tendency to strain crystallize, due presumably to the more orientated short chains serving as nucleating sites. Of course, enhanced strain crystallization generally yields better failure properties. $^{73}$

\section{MISCIBLE BLENDS WITH COMPONENTS HAVING DIFFERENT CROSS-LINK DENSITIES}

The components of an IPN are usually immiscible, with cross-linking used to minimize phase segregation; however, this often does not yield a morphology that is homogeneous on the segmental level. Phase homogeneity of immiscible polymers can be achieved by the use of a co-solvent. A common example of this is hydrogels. ${ }^{74}$ These are dilute aqueous IPNs, which can be engineered to react to changes in $\mathrm{pH}$, temperature, density, the presence of specific chemicals, and so forth. This ability to respond to stimuli, in combination with biocompatibility and a high water content, have 


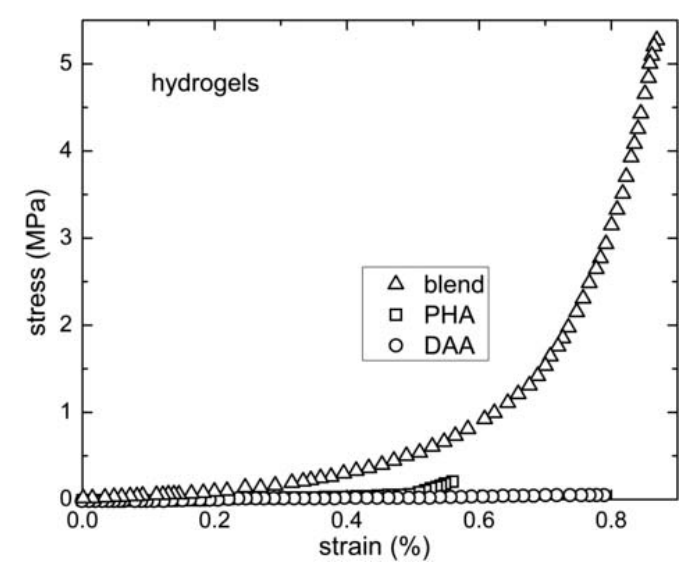

FIG. 11. -Engineering stress versus compressive strain for hydrogels of poly-glycidylmethacrylate-co-hyaluronan (PHA), $\mathrm{N}$-dimethylacrylamide (DAA), and their blend, the last having significantly enhanced mechanical toughness. ${ }^{87}$

led to the use of IPN hydrogels as biomaterials for tissue engineering ${ }^{75-77}$ and for controlled drug delivery. ${ }^{78-81}$ If the two components have very different degrees of cross-linking, hydrogels are referred to as double-network hydrogels (although in the rubber literature and herein, the term double network is applied to a different type of material). Double-network hydrogels are usually produced by sequential free-radical polymerization to form a network within a swollen, previously synthesized network of a different polymer. ${ }^{5}$ When the cross-link densities of the two components are very different, double-network hydrogels exhibit enhanced mechanical properties. ${ }^{82-86}$ For example, Figure 11 shows $^{87}$ the increase in compressive strength of an IPN hydrogel compared with the corresponding single-network hydrogels. Similar results are shown in Figure 12 for an anisotropic double-network hydrogel. ${ }^{88}$ An essential feature is that the respective cross-link densities of the component networks are very different; this is required to achieve improved properties. During deformation, the stiffening effect of short chains may be counterbalanced by softening of the material as it ruptures, ${ }^{25}$ although birefringence measurements indicate that finite extensibility, inherent to such a mechanism, is not the sole contributor to the stiffening. ${ }^{70}$

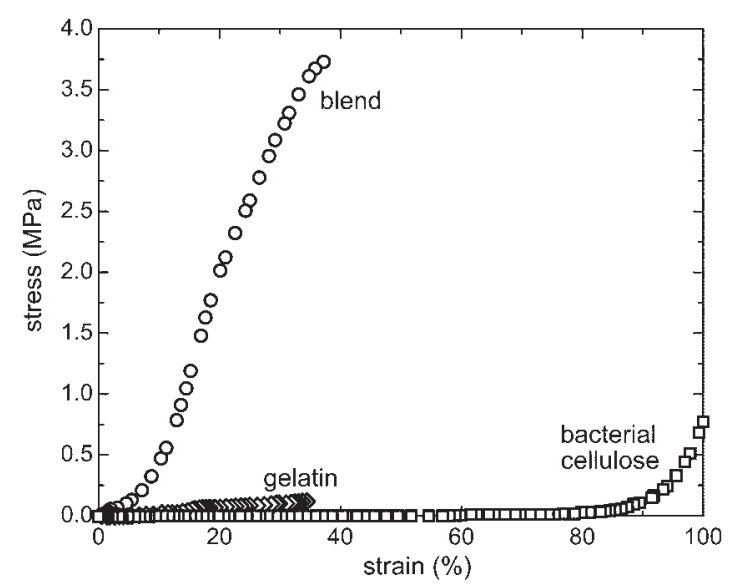

FIG. 12. - Engineering stress versus compressive strain for hydrogels of bacterial cellulose (BC), gelatin (partially hydrolyzed collagen), and their blend. BC consists of planes of a hydrophobic fiber network; the strain was measured perpendicular to these stratified layers. ${ }^{88}$ 


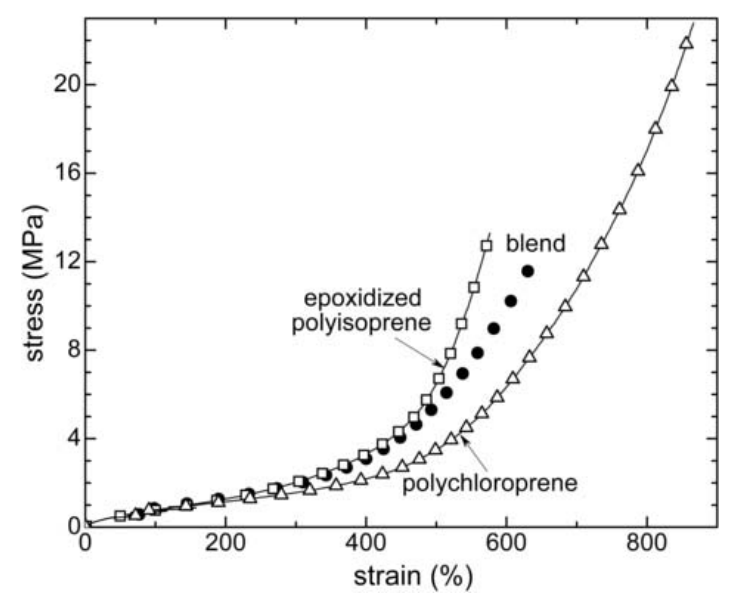

FIG. 13. - Engineering stress-strain data for polychloroprene, epoxidized polyisoprene, and a 50/50 blend; each compound was cured to $90 \%$ of the maximum in their respective rheometer curves. ${ }^{91}$

Hydrogels employ water as a solvent to compatibilize the components, but the requirement for a solvent can be avoided if the polymers comprising the blend are thermodynamically miscible. This means there is no driving force for phase separation, enabling a morphology uniform on the nanometer level to be achieved. Various commercial materials are based on miscible polymer blends, even though a homogeneous morphology does not ensure better mechanical properties. In fact, the properties of a miscible blend are usually an average of those of the pure components. This is illustrated with blends of 1,4-polychloroprene (PC) and epoxidized 1,4-polyisoprene (EPI), which are miscible due to specific interaction of the chlorine atom with the oxirane group. ${ }^{89,90}$ Although some control of the rate and degree of network formation in the components is afforded by the different reaction mechanisms of PC and EPI, only small differences in cross-link density are attained. ${ }^{91}$ Consequently, the blend has mechanical properties intermediate between those of the pure components (Figure 13).

Superior failure properties require a large disparity in the respective cross-link densities of the components, because the operative mechanism is similar to that for bimodal networks but without the requirement of end-linking. Deformation causes the chains of the more cross-linked component to become highly stretched, which confers stiffness to the material; the lightly cross-linked network chains are able to rearrange over sufficient length scales to alleviate local overstressing (inherent to any randomly cross-linked material). This latter spreads the damage zone and suppresses the propagation of rupture nuclei, which would otherwise lead to macroscopic failure.

Using thermodynamically miscible polymers to avoid the requirement for a solvent, there are two approaches to achieve a mixture of a highly cross-linked polymer with one that is lightly or even un-cross-linked. A blend can be prepared from chemically identical polymers that are unreactive to the curing method, except that one component has a few mole percentage repeat units that function as cross-linking sites. An example would be ethylene-propylene copolymer (EPR) mixed with the corresponding random terpolymer of ethylene, propylene, and a few percentage diene (EPDM). In Figure 14 are tensile strength versus modulus data for sulfur-vulcanized EPDM and its blend with EPR, the latter unreactive to sulfur. ${ }^{92}$ This blend is miscible over significant ranges of backbone composition. ${ }^{93}$ The cured material consists of linear EPR chains dispersed in an EPDM network. As seen in the figure, the strength of the blend networks is greater by at least a factor of two than that of the neat EPDM elastomers. 


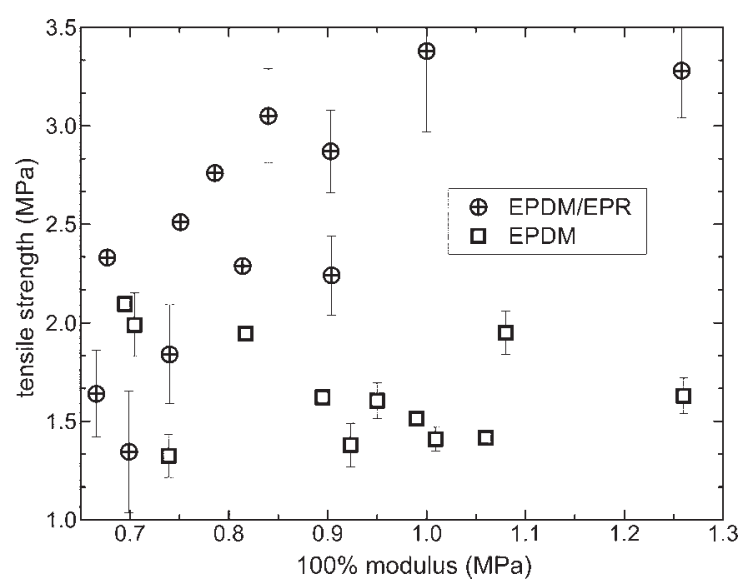

FIG. 14. - Tensile strength versus the engineering stress at $100 \%$ tensile strain for neat EPDM and its blend with EPR. ${ }^{92}$ The blend shows significantly higher tensile strength.

This same approach can be applied to mixtures of polyisobutylene (PIB) with butyl rubber (IIR). Butyl rubber is PIB with a few mole percentage of unsaturated units, usually isoprene. Miscibility of this mixture has been demonstrated through observation of the spontaneous interdiffusion of the two polymers. ${ }^{94}$ PIB does not cross-link, either by sulfur or free radical methods, so the blend is a dispersion of linear PIB chains in an IIR network. These blends have significantly higher strength than neat butyl rubber (Figure 15). ${ }^{94}$

A related method is to use miscible components that have different reactivities to the crosslinking. For example, polyisoprene (IR) and polyvinylethylene (PVE) are thermodynamically miscible, ${ }^{95-98}$ with the system exhibiting a lower critical solution temperature (LCST) around 160 ${ }^{\circ} \mathrm{C} .{ }^{99}$ Heterogeneous networks can be produced because the tertiary vinyl carbons of PVE do not sulfurize, ${ }^{100}$ whereas the IR readily vulcanizes. However, if the curing is carried out at elevated temperatures, above the LCST (which is lowered by the chemical changes effected by the cross-

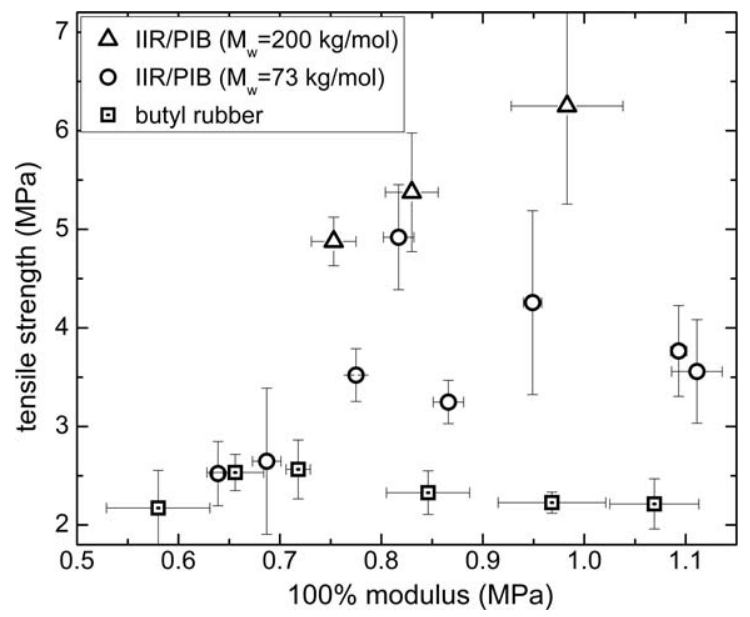

FIG. 15. - Tensile strength for neat butyl rubber and two blends with polyisobutylenes having the indicated molecular weights, as a function of the modulus at $100 \%$ tensile strain. ${ }^{94}$ The blends have higher tensile strengths, more so for the higher-molecular-weight PIB. 


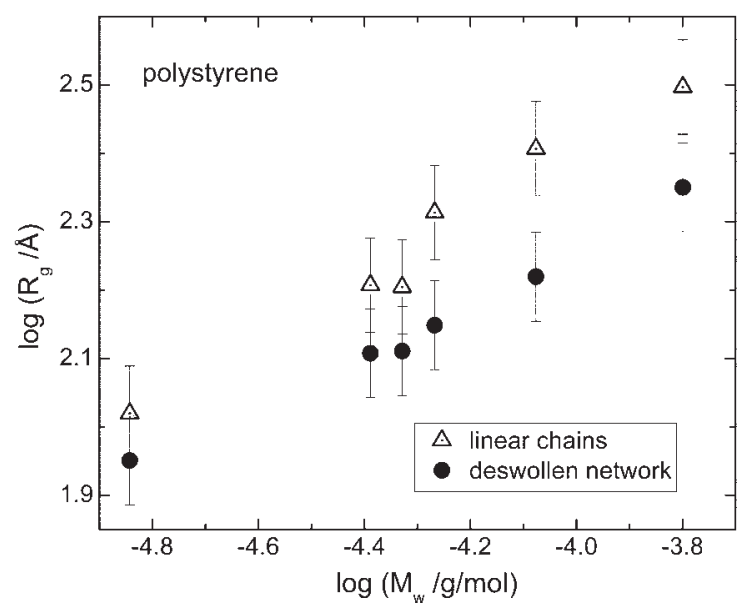

FIG. 16. - Radius of gyration of deuterated polystyrene chains dispersed in a melt (triangles) and in a network cross-linked while swollen $\left(V / V_{x}=10\right)$ and then dried (circles). ${ }^{104}$ The abscissa is the molecular weight of either the linear polystyrene or the network chains. Removal of the solvent causes collapse of the network chains, yielding smaller coil dimensions than for a conventional network.

linking reaction), the components phase segregate doing curing. Consequently, a homogeneous material is not obtained, and thus the mechanical properties are not improved. ${ }^{101}$ To avoid this problem, radiation cross-linking at ambient temperature can be used, taking advantage of the greater sensitivity of the PVE to free radical reaction. By this means, the homogeneous morphology is retained, although efforts to date have not yielded the degree of cross-link disparity necessary for optimal properties. ${ }^{102}$

\section{E. DESWOLLEN NETWORKS}

Cross-linking a polymer containing diluent and then subsequently removing the liquid has two effects: the size of the network chains is reduced, and they have fewer trapped entanglements. When

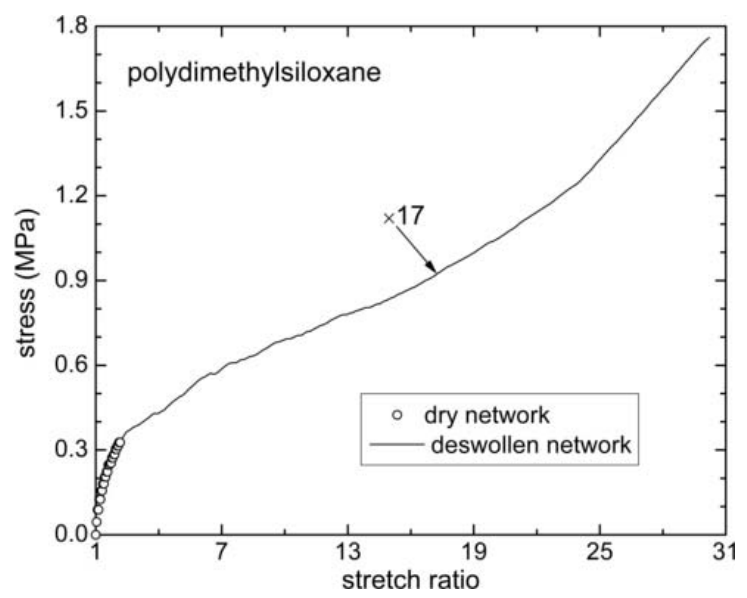

FIG. 17. -Engineering stress versus stretch ratio for a conventional network (circles) and a network formed in solution ( $V /$ $V_{x}=1.25$ ) and then dried (line). ${ }^{107}$ Data for the latter were scaled as indicated to superpose at low strains on the other material. 


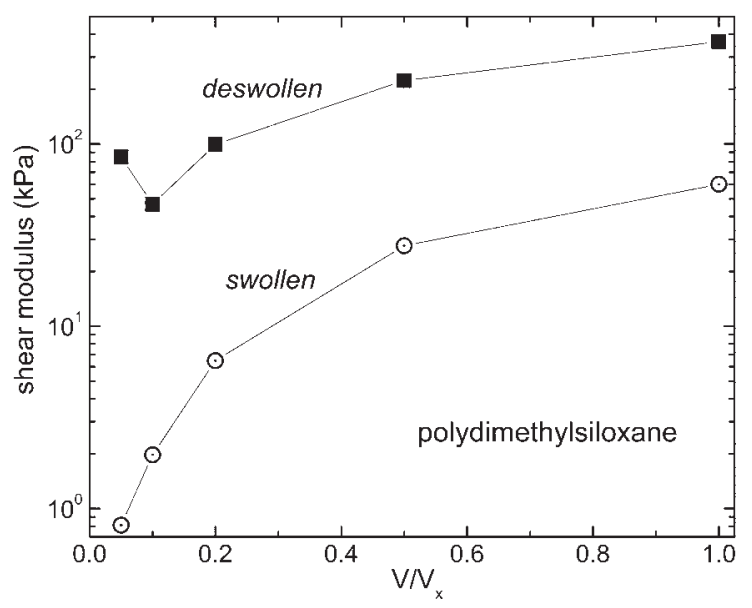

FIG. 18. - Comparison of the modulus of PMDS cross-linked at the indicated volume fraction measured before (circles) and after (squares) deswelling. ${ }^{108}$ The greater density of chains in the dry network exerts a larger effect on the modulus than that due to the collapse of the chains resulting from deswelling.

this dried network is deformed, the stretch ratio of the chains, $\lambda_{0}$, is less than the macroscopic stretch ratio, $\lambda$, according to

$$
\lambda_{0}=\left(\frac{V}{V_{\mathrm{x}}}\right)^{1 / 3} \lambda
$$

where $V_{\mathrm{x}}$ and $V$ are the respective volumes during cross-linking and the subsequent deformation. For substantial swelling (large $V_{x}$ ), the deswelling after cross-linking results in a highly compacted or "supercoiled" network. ${ }^{103}$ This can be seen in the value of the radius of gyration of polystyrene deswollen $\left(V / V_{x}=0.1\right)$ after varying degrees of cross-linking (Figure 16). ${ }^{104}$ The reduction in trapped entanglements lowers the modulus by a factor approximately inversely proportional to the volume fraction of chains during the cross-linking. ${ }^{105}$

This combination of a highly compressed network of less entangled chains causes deswollen networks to have a low modulus and remarkably large strains to failure; these properties are referred to as "super-elasticity." 105,106 In Figure 17, stress-strain curves are compared for conventional and deswollen networks. ${ }^{107}$ In Figure 18, the modulus of deswollen networks is plotted versus $V_{x}$. Note there is a minimum in the data, ascribed to the greater packing of the compacted chains, which increases their density (about $15 \%$ for $V_{x}>>V^{108}$ ). More chains per unit area contribute to a higher modulus, countervailing the effect of the collapsed configuration and lower entanglements. Another consequence of a compact chain structure is slower thermal crystallization of unoriented deswollen networks. ${ }^{109}$

\section{SUMMARY}

Different approaches to obtaining elastomers with improved strength and other failure properties have been reviewed. Some methods require the use of solvents and therefore have narrow applicability (hydrogel IPNs for bioengineering) or are of academic interest (deswollen networks). Bimodal networks require end-linking and thus are not generally useful, and the requirement for simultaneous polymerization and cross-linking severely restricts the utility of IPNs. Notwithstanding these limitations, results from such materials demonstrate concepts that 
might be exploited by alternative means. Double networks and blends of miscible polymers with disparate cross-linking are two approaches that have the advantage of employing commonly used polymers. There are additional processing steps required to produce double networks, and whether these can be done efficiently and cost-effectively is uncertain. The miscible blend route to alternative networks can be implemented with existing products, for example, by substituting a portion of EPDM or butyl rubber with their respective nonreactive analogs. The issue therein is whether the effect on other properties, notably hysteresis, is acceptable. Notwithstanding the practical difficulties of alternative network structures, it is gratifying that almost one and threequarter centuries after Charles Goodyear vulcanized natural rubber, the cross-linking of rubber continues to be an exciting research topic with technological promise.

\section{ACKNOWLEDGEMENT}

This work was supported by the Office of Naval Research.

\section{REFERENCES}

${ }^{1}$ I. S. Choi, C. M. Roland, and L. C. Bissonnette, RubBer CHEM. TeCHNOL. 67, 892 (1994).

${ }^{2}$ W. J. MacKnight and R. D. Lundberg, RubBer CHEM. TeChNOL. 57, 652 (1984).

${ }^{3}$ P. Antony and S. K. De, J. Macromol. Sci. Polym. Rev. C41, 41 (2001).

${ }^{4}$ W. V. Mars, RubBer CHEM. TECHNOL. 84, 178 (2011).

${ }^{5}$ J. P. Gong, Y. Katsuyama, T. Kurokawa, and Y. Osada, Adv. Matl. 15, 1155 (2003).

${ }^{6}$ K. Ito, Curr. Opin. Sol. State Mat. Sci. 14, 28 (2010).

${ }^{7}$ L. H. Sperling, Interpenetrating Polymer Networks and Related Materials, Plenum, New York, 1981.

${ }^{8}$ Advances in Interpenetrating Polymer Networks, Vol. 1, D. Klempner and K. C. Frisch, Eds., Technomic, Lancaster, 1989.

${ }^{9}$ IPNs around the World: Science and Engineering, S. C. Kim and L. H. Sperling, Eds., Wiley, Chichester, 1997.

${ }^{10}$ N. Gupta and A. K. Srivastava, Polym. Int. 35, 109 (1994).

${ }^{11}$ B. Suthar, H. X. Xiao, D. Klempner, and K. C. Frisch, Polym. Adv. Technol. 7, 221 (1996).

${ }^{12}$ J. M. M. Dueñas and J. L. G. Ribelles, J. Therm. Anal. Calor. 72, 695 (2003).

${ }^{13}$ N. R. Manoj, R. D. Raut, P. Sivaraman, D. Ratna, and B. C. Chakraborty, J. Appl. Polym. Sci. 96, 1487 (2005).

${ }^{14}$ R. P. Burford, M. G. Markotsis, and R. B. Knott, Physica B 385-386, 766 (2006).

${ }^{15}$ T. Tamai, A. Imagawa, and Q. Tran-Cong, Macromolecules 27, 7486 (1994).

${ }^{16}$ X. Ramis, A. Cadenato, and J. M. Morancho, Polymer 42, 9469 (2001).

${ }^{17}$ F. Vidal, O. Fichet, J. Laskar, and D. Teyssié, Polymer 47, 3747 (2006).

${ }^{18}$ N. V. Babkina, Y. S. Lipatov, T. T. Alekseeva, L. A. Sorochinskaya, and Y. I. Datsyuk, Polym. Sci. Ser. A 50, 798 (2008).

${ }^{19}$ Sound and Vibration Damping with Polymers, ACS Symp. Ser. 424, R. D. Corsaro and L. H. Sperling, Eds., 1990.

${ }^{20}$ D. I. G. Jones, Handbook of Viscoelastic Vibration Damping, Wiley Interscience, New York, 2001.

${ }^{21}$ R. B. Fox, J. P. Armistead, C. M. Roland, and D. J. Moonay, J. Appl. Polym. Sci. 41, 1281 (1990).

${ }^{22}$ K. C. Frisch, D. Klempner, S. Migdal, H. L. Frisch, and H. Ghiradella, Polym. Eng. Sci. 14, 76 (1974).

${ }^{23}$ Y. M. Lee, Y. S. Yang, and L. J. Lee, Polym. Eng. Sci. 27, 716 (1987).

${ }^{24}$ C. Chunming, X. Chuanxi, Y. Jian, and D. Lijie, J. Wuhan Univ. Technol. Mater. Sci. Ed. 23, 50 (2008).

${ }^{25}$ X. Zhao, J. Mech. Phys. Solids 60, 319 (2012).

${ }^{26}$ S. M. Ha, W. Yuan, Q. B. Pei, R. Pelrine, and S. Stanford, Adv. Mater. 18, 887 (2006).

${ }^{27}$ S. Granick and J. D. Ferry, Macromolecules 16, 39 (1983).

${ }^{28}$ W. Batsberg and O. Kramer, J. Chem. Phys. 74, 6507 (1981). 
${ }^{29}$ A. V. Tobolsky, Y. Takahashi, and S. Naganuma, Polym. J. 3, 60 (1972).

${ }^{30}$ K. T. Gillen, Macromolecules 21, 442 (1988).

${ }^{31}$ J. Scanlan and W. F. Watson, Trans. Far. Soc. 54, 740 (1958).

${ }^{32}$ L. Mandelkern, D. E. Roberts, A. F. Diorio, and A. S. Posner, J. Am. Chem. Soc. 81, 4148 (1959).

${ }^{33}$ R. A. M. Hikmet, J. Lub, and P. M. Vanderbrink, Macromolecules 25, 4194 (1992).

${ }^{34}$ W. F. Reichert, D. Goritz, and E. J. Duschl, Polymer 34, 1216 (1993).

${ }^{35}$ C. M. Roland, Viscoelastic Behavior of Rubbery Materials, Oxford, Oxford University Press, 2011.

${ }^{36}$ W. Ren, P. J. McMullan, and A. C. Griffin, Macromol. Chem Phys. 209, 1896 (2008).

${ }^{37}$ R. D. Andrews, A. V. Tobolsky, and E. E. Hanson, J. Appl. Phys. 17, 352 (1946).

${ }^{38}$ T. Termonia, Macromolecules 23, 1976 (1990).

${ }^{39}$ L. G. Baxandall and S. F. Edwards, Macromolecules 21, 1763 (1988).

${ }^{40}$ B. Meissner and L. Matejka, Polymer 44, 4611 (2003).

${ }^{41}$ P. H. Mott and C. M. Roland, Macromolecules 29, 6941 (1996).

${ }^{42}$ D. E. Hanson and C. M. Roland, J. Polym. Sci. Pt. B-Polym. Phys. 48, 1795 (2010).

${ }^{43}$ P. G. Santangelo and C. M. Roland, RubBER CHEM. TECHNOL. 67, 359 (1994).

${ }^{44}$ P. G. Santangelo and C. M. Roland, RuBber CHEM. TECHNOL. 68, 124 (1995).

${ }^{45}$ G. R. Hamed and M. Y. Huang, RubBer CHEM. TECHNOL. 71, 846 (1998).

${ }^{46}$ A. Greene, K. J. Smith, and A. Ciferri, Trans. Far. Soc. 61, 2772 (1965).

${ }^{47}$ C. M. Roland and M. L. Warzel, RubBer CHEM. TeChNOL. 63, 285 (1990).

${ }^{48}$ C. V. Marykutty, G. Mathew, and S. Thomas, Rubber ChEM. TeChNOL. 64, 790 (1991).

${ }^{49}$ P. H. Mott and C. M. Roland, Macromolecules 33, 4132 (2000).

${ }^{50}$ C. M. Roland and K. L. Peng, Rubber ChEM. TeChNOL. 64, 790 (1991).

${ }^{51}$ J. Wang, G. R. Hamed, K. Umetsu, and C. M. Roland, RubBER CHEM. TECHNOL. 78, 76 (2005).

${ }^{52}$ P. G. Santangelo and C. M. Roland, RuBber CHEM. TECHNOL. 76, 892 (2003).

${ }^{53}$ G. J. Lake and P. B. Lindley, J. Appl. Polym. Sci. 10, 343 (1966).

${ }^{54}$ J. E. Mark, Macromol. Symp. 191, 121 (2003).

${ }^{55}$ V. K. Soni and R. S. Stein, Macromolecules 23, 5257 (1990).

${ }^{56}$ W. Wu, L. D. Coyne, L. Jong, A. Hanyu, and R. S. Stein, Macromolecules 23, 351 (1990).

${ }^{57}$ H. Oikawa, Polymer 33, 1116 (1992).

${ }^{58}$ B. D. Viers and J. E. Mark, J. Mac. Sci. Pure Appl. Chem. 44, 131 (2007).

${ }^{59}$ J. U. Sommer and S. Lay, Macromolecules 35, 9832 (2002).

${ }^{60}$ K. Urayama, T. Kawamura, Y. Hirata, and S. Kohjiya, Polymer 39, 3827 (1998).

${ }^{61}$ A. L. Larsen, P. Sommer-Larsen, and O. Hassager, e-Polymers 050 (2004).

${ }^{62}$ T. L. Smith, B. Haidar, and J. L. Hedrick, RubBER CHEM. TECHNOL. 63, 256 (1990).

${ }^{63}$ J. E. Mark and M. Y. Tang, J. Polym. Sci. Polym. Phys. Ed. 22, 1849 (1984).

${ }^{64}$ G. B. Shah, J. Appl. Polym. Sci. 94, 1719 (2004); e-Polymers 113 (2007).

${ }^{65}$ F. Bueche, Physical Properties of Polymers, Wiley-Interscience, New York, 1962.

${ }^{66}$ J. E. Mark, RUBBER CHEM. TECHNOL. 72, 465 (1999).

${ }^{67}$ A. Hanyu and R. S. Stein, Makromol. Chem. Macromol. Symp. 45, 189 (1991).

${ }^{68}$ G. A. Holmes and A. Letton, Polym. Eng. Sci. 34, 1635 (1994).

${ }^{69}$ N. C. B. Tan, B. J. Bauer, J. Plestil, J. D. Barnes, D. Liu, L. Matejka, K. Dusek, and W. L. Wu, Polymer 40, 4603 (1999).

${ }^{70}$ D. Viers and J. E. Mark, J. Inorgan. Organometallic Polym Matl. 17, 283 (2007).

${ }^{71}$ C. C. Sun and J. E. Mark, J. Polym. Sci. Polym. Phys. Ed. 25, 2073 (1987).

${ }^{72}$ A. Hanyu and R. S. Stein, Makromol. Chem. Macromol. Symp. 45, 189 (1991).

${ }^{73}$ I. S. Choi and C. M. Roland, RubBER CHEM. TECHNOL. 70, 202 (1997). 
${ }^{74}$ D. Myung, D. Waters, M. Wiseman, P. E. Duhamel, J. Noolandi, C. N. Ta, and C. W. Frank Polym. Adv. Technol. 19, 647 (2008).

${ }^{75}$ D. Myung, W. Koh, J. Ko, Y. Hu, M. I. Carrasco, J. Noolandi, C. N. Ta, and C. W. Frank, Polymer 48, 5376 (2007).

${ }^{76}$ E. F. Irwin, K. Saha, M. Rosenbluth, L. J. Gamble, D. G. Castner, and K. E. Healy, J. Biomatl. Sci. Polym. Ed. 19, 1363 (2008).

${ }^{77}$ Y. Liu and M. Chan-Park, Biomaterials 30, 186 (2009).

${ }^{78}$ Z. S. Akdemir and N. Kayaman-Apohan, Polym. Adv. Technol. 18, 932 (2007).

${ }^{79}$ Q. Wang, S. Li, Z. Wang, H. Liu, and C. Li, J. Appl. Polym. Sci. 111, 1417 (2008).

${ }^{80}$ H. Ajiro, Y. Takemoto, and M. Akashi, Chem. Lett. 38, 368 (2009).

${ }^{81}$ Y. Yue, P. Wang, Q. Chen, and X. G. Liu, J. Appl. Polym. Sci. 112, 2261 (2009).

${ }^{82}$ J. Kopecek and J. Yang, Polym. Int. 56, 1078 (2007).

${ }^{83}$ D. Myung, D. Waters, M. Wiseman, P.-E. Duhamel, J. Noolandi, C. N. Ta, and C. W. Frank, Polym. Adv. Technol. 19, 647 (2008).

${ }^{84}$ L. K. Kostanski, R. X. Huang, C. D. M. Felip, and R. Ghosh, J. Biomatl. Sci. Polym. Ed. 20, 271 (2009).

${ }^{85}$ H. Tsukeshiba, M. Huang, Y.-H. Na, T. Kurokawa, R. Kuwabara, Y. Tanaka, H. Furukawa, Y. Osada, and J. P. Gong, J. Phys. Chem. B 109, 16304 (2005).

${ }^{86}$ T. Nakajima, T. Kurokawa, S. Ahmed, W.-L. Wu, and J. P. Gong, Soft Matter 9, 1955 (2013).

${ }^{87}$ L. Weng, A. Gouldstone, Y. Wu, and W. Chen, Biomaterials 29, 2153 (2008).

${ }^{88}$ M. A. Haque, T. Kurokawa, and J. P. Gong, Polymer 53, 1805 (2012).

${ }^{89}$ J. B. Nagode and C. M. Roland, Polymer 32505 (1991).

${ }^{90}$ J. H Walton, J. B. Miller, and C. M. Roland, J. Poly. Sci. Poly. Phys. Ed. 30527 (1992).

${ }^{91}$ Y. T. Vu and C. M. Roland, Unpublished work, 2004.

${ }^{92}$ G. S. Buckley, D. M. Fragiadakis, and C. M. Roland, RubBer CHEM. TECHNOL. 84, 520 (2011).

${ }^{93}$ H. G. Kikland and M. Van Duin, RubBer ChEM. TECHNOL. 76, 495 (2003).

${ }^{94}$ C. B. Giller and C. M. Roland, Macromolecules, in press.

${ }^{95}$ G. M. Bartenev and G. S. Kongarov, RUBBer CHEM. TECHNOL. 36, 668 (1963).

${ }^{96}$ R. E. Cohen, Adv. Chem. Series 193, 489 (1982).

${ }^{97}$ C. M. Roland, Macromolecules 20, 2557 (1987).

${ }^{98}$ S. Kawahara, S. Akiyama, and A. Ueda, Polymer J. 21, 221 (1989).

${ }^{99}$ D. W. Tomlin and C. M. Roland, Macromolecules 25, 2994 (1992).

${ }^{100}$ A. Y. Coran, in Science and Technology of Rubber, F. R. Ehrich, Ed., Academic Press, New York, 1978.

${ }^{101}$ J. Wang and C. M. Roland, Polymer 46, 4160 (2005).

${ }^{102}$ C. B. Giller and C. M. Roland, Unpublished work 2013.

${ }^{103}$ J. J. Hermans, J. Polym. Sci. 59, 191 (1962).

${ }^{104}$ J. Bastide, in Physics of Finely Divided Matter, Springer Proceedings of Physics, Vol. 5, N. Boccara and M. Daoud, Eds., Springer, Berlin, 1985.

${ }^{105}$ S. P. Obukhov, M. Rubinstein, and R. H. Colby, Macromolecules 27, 3191 (1994).

${ }^{106}$ K. Sivasailam and C. Cohen, J. Rheol. 44, 897 (2000).

${ }^{107}$ K. Urayama and S. Kohjiya, Eur. Phys. J. B 2, 75 (1998).

${ }^{108}$ V. G. Vasiliev, L. Z. Rogovina, and G. L. Slonimsky, Polymer 26, 1667 (1985).

${ }^{109}$ K. Urayama, K. Yokoyama, and S. Kohjiya, Polymer 41, 3273 (2000). 